\title{
InFORMAL Insurance IN SOCIAL NeTwORKS
}

\author{
Francis Bloch \\ GREQAM, Université de la Méditerranée and Warwick University \\ Garance Genicot \\ Georgetown University \\ and \\ Debraj Ray \\ New York University and Instituto de Análisis Económico (CSIC) \\ July 2005, revised September 2006
}

\begin{abstract}
This paper studies bilateral insurance schemes across networks of individuals. While transfers are based on social norms, each individual must have the incentive to abide by those norms, and so we investigate the structure of self-enforcing insurance networks. Network links play two distinct and possibly conflictual roles. First, they act as conduits for transfers. Second, they act as conduits for information. These features affect the scope for insurance, as well as the severity of punishments in the event of noncompliance. Their interaction leads to a characterization of stable networks as networks which are suitably "sparse", the degree of sparseness being related to the length of the minimal cycle that connects any triple of agents. As corollaries, we find that both "thickly connected" networks (such as the complete graph) and "thinly connected" networks (such as trees) are likely to be stable, whereas intermediate degrees of connectedness jeopardize stability. Finally, we study in more detail the notion of networks as conduits for transfers, by simply assuming a punishment structure (such as autarky) that is independent of the precise architecture of the network. This allows us to isolate a bottleneck effect: the presence of certain key agents who act as bridges for several transfers. Bottlenecks are captured well in a feature of trees that we call decomposability, and we show that all decomposable networks have the same stability properties and that these are the least likely to be stable.
\end{abstract}

JEL Classification Numbers: D85, D80, 012, Z13

Keywords: social networks, reciprocity networks, norms, informal insurance.

An earlier draft was written while Genicot and Ray were visiting the London School of Economics; we thank the LSE for their hospitality. Ray is grateful for funding from the National Science Foundation under grant no. 0241070. We are grateful for comments by seminar participants at Barcelona, Cergy, CORE, Essex, Helsinki LSE/UCL, Oxford and University of Marylandand and audiences at the NEUDC in Montréal, the AEA Meetings in Philadelphia, the SED in Budapest, conferences on networks and coalitions in Vaxholm and Guanajato, and the Conference in tribute to Jean-Jacques Laffont in Toulouse. Contact the authors at francis.bloch@univmed.fr, gg58@georgetown.edu, and debraj.ray@nyu.edu. 


\section{INTRODUCTION}

This paper studies networks of informal insurance. Such networks exist everywhere, but especially so in developing countries and in rural areas where credit and insurance markets are scarce and income fluctuations are endemic. Yet it is also true that everybody does not enter into reciprocal insurance arrangements with everybody else, even in relatively small village communities. A recent empirical literature (see for instance Fafchamps (1992), Fafchamps and Lund (2003), and Murgai, Winters, DeJanvry and Sadoulet (2002)) shows that insurance schemes often takes place within subgroups in a community. One obvious reason for this is that everyone may not know one another at a level where such transactions become feasible. A community — based on friends, extended family, kin or occupation - comes first, insurance comes later. This is the starting point of our paper. ${ }^{1}$

Once this view is adopted, however, it is clear that an "insurance community" is not a closed multilateral grouping. $A$ may insure with $B$, and $B$ with $C$, but $A$ and $C$ may have nothing to do with each other. The appropriate concept, then, is one of an insurance network. Empirically, such networks have attracted attention and have recently been mapped to some extent: for instance, Stack (1974), Wellman (1992), de Weerdt (2002), Dercon and de Weerdt (2000), and Fafchamp and Gubert (2004) reveal a complex architecture of risk-sharing networks.

The very idea of an insurance network rather than a group suggests that our existing notion of insurance as taking place within an multilateral "club" of several people may be misleading. Of course, such clubs may well exist, but a significant segment of informal insurance transactions is bilateral. $A$ and $B$ will have their very own history of kindness, reciprocity or betrayal. In these histories, either party may have been have cognizant of (and taken into account) her partner's obligations to (or receipts from) a third individual, but the fundamental relationship is nevertheless bilateral.

A principal aim of our paper is to build a model of risk-sharing networks which captures this feature. A prior relationship is used to define the network. Once in place, both insurance and the transfer of information is limited by this network. Links can be broken, if people have "betrayed" their relationship with an unkept promise to insure each other, but new links cannot be formed. While there are merits to an alternative exercise in which new links can be deliberately formed, there is also much to be gained from studying a view in which existing links are nonstrategic, and so we view these two frameworks as complementary. ${ }^{2}$

In the model studied here, only "directly linked" agents in some given network make transfers to each other, though they are aware of the (aggregate) transfers each makes to others. We view insurance as being based on internalized norms regarding mutual help. A bilateral insurance norm between two linked agents specifies consumptions for every linked pair of individuals, as a function of various observables such as their identities, the network component they belong to, their income realizations and the transfers made to or received from other agents. These transfers are taken as given by the linked pair, but are obviously endogenous for society as

\footnotetext{
${ }^{1}$ As Genicot and Ray $(2003,2005)$ have argued, there may also be strategic reasons for limited group formation. Genicot and Ray build on a large literature which studies insurance schemes with self-enforcement constraints; see, e.g., Posner (1980), Kimball (1988), Coate and Ravallion (1993), Kocherlakota (1996), Kletzer and Wright (2000) and Ligon, Thomas and Worrall (2002)).

${ }^{2}$ In research that has recently come to our attention, Bramoullé and Kranton (2005) study the formation of insurance networks under the assumption of equal division and perfect enforcement. By contrast, our paper studies a family of insurance schemes — including equal division — in an explicit context of self-enforcement, but assumes that the network is given for exogenous reasons such as friendship, family, or social contacts. In our model, links can be broken but new links can never be formed.
} 
a whole. We therefore introduce the notion of a consistent consumption allocation, one that allocates consumptions to everyone for each realization of the state, and which implicitly agrees with the bilateral norm for every linked pair.

With this setup as background, the paper then studies the stability of insurance networks, explicitly recognizing the possibility that the lack of commitment may destabilize insurance arrangements. Thus a consumption allocation cannot only be consistent (with the underlying norm); it must also be self-enforcing.

But precisely what does self-enforcement entail? In the "group-based" insurance paradigm, a natural supposition is that a deviating individual is thereafter excluded from the group, and that is what the bulk of the literature assumes. Yet if arrangements are fundamentally bilateral, this sort of exclusion needs to be looked at afresh. If $A$ deviates from some arrangement with $B$, we take it as reasonable that $B$ refuses to engage in future dealings with $A .^{3}$ The payoff consequences of this refusal may be taken to be the weakest punishment for $A$ 's misbehavior. But the punishment may conceivably be stronger: $B$ might "complain" to third parties. If such parties are linked directly to $A$ they, too, might break their links (such breakage would be sustained by the usual repeated-game style construction that zero interaction always constitutes an equilibrium). To go further, third parties might complain to fourth parties, who in turn might break with $A$ if they are directly linked, and so on. Such complaints will travel along a "communication network" which in principle could be different from the network determining direct transfers, but in this paper we take the two networks to be the same. ${ }^{4}$ If all agents are indirectly connected in this way, then the limiting case in which all news is passed on - and corresponding action taken - is the one of full exclusion typically assumed in the literature. We propose to examine the intermediate cases.

Our analysis highlights two forces in the relation between the architecture of the network and the stability of insurance schemes: an informational effect that determines the capacity of the network to punish deviants, and a transit or bottleneck effect that arises from the restriction that transfers must only take place between linked agents.

We first study the informational effect. To rule out the short-term features we deliberately focus on discount factors close to unity. The first principal result (Proposition 3) of the paper provides a full characterization of those insurance networks that satisfy the self-enforcement constraint for different "levels" of communication. By "level" we refer to the number of rounds $q$ of communication (and consequent retribution) that occur following a deviation: for instance, if the immediate victim talks to no one else, $q=0$, if she talks to her friends who talk to no one else, then $q=1$, and so on. For any such $q$, we provide a characterization of those network architectures that are stable under the class of monotone insurance norms, those in which the addition of new individuals to a connected component by linking them to one member increases that member's payoff. The characterization involves a particular property of networks. As an implication, for any $q$, typically both thinly and thickly connected networks are most conducive to stability; intermediate degrees of connection are usually unstable.

To obtain an intuitive feel for this implication, imagine that $q$ is small. Nevertheless, if the network is very thin the miscreant may still be effectively cut off (and thereby adequately punished) even though the accounts of his deviation do not echo fully through the network (he

\footnotetext{
${ }^{3}$ Of course, issues of renegotiation might motivate a reexamination of even this assumption, but we do not do that here.

${ }^{4}$ Certainly, it is perfectly reasonable to suppose that the communication network is a superset of the transfers network. So the assumption - that we make to take a first tractable step in this area - is that all pairs who can talk can also make transfers.
} 
was tenuously connected anyway). On the other hand, if the network is fully connected a single round of complaints to third parties is also enough to punish the miscreant, because there will be many such "third parties" and they will all be connected to him. It is precisely in networks of intermediate density that the deviant may be able to escape adequate punishment.

This suggest a $U$-shaped relationship between network density and stability for intermediate level of communication. To be sure, this is not a one-to-one relationship as networks of very different architecture have the same average density. However, by simulating many networks of a given density and assessing their stability, we can illustrate this $U$-shaped relationship. Similarly, we show a positive relationship between clustering and stability. This is particularly useful as the density and clustering coefficient (concepts that we will define precisely later) are basic characteristics of networks used in the social network literature (Wasserman and Faust (1994)).

Next, we study the transit or bottleneck effect, and so consider discount factors which are not close to unity. Assessing the stability of mutual insurance schemes in such contexts is a difficult task. We do so assuming a specific risk-sharing norm: equal sharing, in which all pairs of agents divide their income (net of third-party obligations but including third-party transfers) equally at every state.

As transfers can only flow along links in the network, the consistent transfer scheme associated with such a norm may effectively require excessive reliance on a particular "bottleneck" agent, which in turn increases her short term incentive to deviate from the scheme. If we abstract from the subtleties of the information effect by assuming that all deviants are punished by full exclusion (say $q$ is large), then this effect becomes particularly clear, as the post-deviation continuation value for every agent is the same. In this situation, one can identify — for any network the "bottleneck" agent by simply looking at the maximal short-term incentive to deviate. The enforcement constraint faced by this bottleneck agent defines the stability of the entire network. In Proposition 4, we isolate a class of "decomposable" networks (which includes all stars and lines) for which the bottleneck effect is identical, and hence stability conditions are identical. Moreover, we show that decomposable networks are the networks for which the bottleneck effects are the most acute. The addition of new links can only relax the bottleneck effect, as new links can be used to reroute transfers at every state. It follows that adding links only improves the stability of the network, and that the complete network is stable for lower values of the discount factor than any other network.

However, this finding is for the case of strong punishments, in which all agents are punished by full exclusion. For weaker punishment schemes, we show that a higher density in a network has an ambiguous effect. On the one hand, it reduces the bottleneck effects, thereby helping stability, but, as seen earlier, it also reduces the potential punishment a deviant would suffer which hurts stability.

We believe that this paper represents a first step in the study of self-enforcing insurance schemes in networks. In taking this step, we combine methods from the basic theory of repeated games, which are commonly used for models of informal insurance, with the more recent theories of networks. It appears that this combination does yield some new insights, principal among them being our characterization of stable networks. However, it is only fair to add that we buy these insights at a price. For instance, it would be of great interest to study the case in which the aggregate of third-party transfers is not observable. This would introduce an entirely new set of incentive constraints, and is beyond the scope of the present exercise.

Qualifications notwithstanding, our findings contribute to a recent and growing literature on the influence of network structures in economics. See for instance, Calvó-Armengol and 
Jackson (2004) on labor markets, Goyal and Joshi (2003) on networks of cost-reducing alliances, Bramoullé and Kranton (2004) on public goods, Tesfatsion (1997, 1998) and Weisbuch, Kirman and Herreiner (2000) on trading networks, Fafchamps and Lund (1997) on insurance, Conley and Udry (2002), Chatterjee and Xu (2004) and Bandiera and Rasul (2002) on technology adoption, and Kranton and Minehart $(2000,2001)$ and Wang and Watts (2002) on buyer-seller networks.

\section{Transfer Norms in Insurance Networks}

2.1. Endowments and Preferences. We consider a community of individuals occupying different positions in a social network (see below). At each date, a state of nature $\theta$ (with probability $p(\theta))$ is drawn from some finite set $\Theta$. The state determines a strictly positive endowment $y_{i}$ for agent $i$. Denote by $\mathbf{y}(\theta)$ the vector of income realizations for all agents. Assume that every possible inter-individual combination of (a finite set of) outputs has strictly positive probability. [This condition guarantees, in particular, that outputs are not perfectly correlated.]

Agent $i$ is endowed with a smooth, increasing and strictly concave von Neumann-Morgenstern utility $u_{i}$ defined over consumption, and a discount factor $\delta_{i} \in(0,1)$. Individual consumption will not generally equal individual income as agents will make transfers to one another. However, we assume that the good is perishable and that the community as a whole has no access to outside credit, so aggregate consumption cannot exceed aggregate income at any date.

2.2. Networks. Agents interact in a social network. Formally, this is a graph $g$ - a collection of pairs of agents - with the interpretation that the pair $i j$ belongs to $g$ if they are directly linked. In this paper, a bilateral link is a given: it comes from two individuals getting to know each other for reasons exogenous to the model. While such links may be destroyed (for instance, due to an unkept promise), no new links can be created.

Note that two individuals are connected in a network if they are directly or indirectly linked, and the components of a network are the largest subsets of connected individuals. For any component $h$ of a graph $g$, we denote by $N(h)$ the set of agents in component $h$.

For our purposes, a link between $i$ and $j$ means two things. First, it means that $i$ and $j$ can make transfers to each other. Second, it is a possible avenue for the transmission of information (more on this below).

2.3. Bilateral Norms. In sharp contrast to existing literature, we take a decentralized view of insurance. Any two linked individuals may insure each other. This implies some degree of insurance for larger groups, but no deliberate scheme exists for such groups. To be sure, transfers from or to an individual must take into account what her partner is likely to receive from (or give to) third parties. In many situations this is easier said than done. Such transfers may not be verifiable, and in any case all transfers are made simultaneously. As a first approximation we assume that for every linked pair, third-party transfers are verifiable ex post, and that the values of such transfers inform the bilateral dealings of the pair in a way made precise below.

In short, for a linked pair $i j$, state-contingent income vectors $\left(\mathbf{y}_{i}\right.$ and $\left.\mathbf{y}_{j}\right)$ as well as third-party transfers by each agent $\left(\mathbf{z}_{i}\right.$ and $\left.\mathbf{z}_{j}\right)$ are observed and conditioned upon. ${ }^{5}$ These latter variables are endogenous; their exact form will be pinned down in society-wide equilibrium.

\footnotetext{
${ }^{5}$ Clearly, the third party obligations of agents $i$ and $j$ depend on the specific pair of agents considered. Because we focus on one specific pair of agents, we do not need to take this dependency explicitly into account in our notation.
} 
For instance, consider a three person network in which $B$ is linked to both $A$ and $C$. Use the notation $x_{i j}$ to denote the transfer, positive or negative, from $j$ to $i$. Figure 1 illustrates transfers that equate consumptions in a state in which $B$ and $C$ 's income is 1 while $A$ 's income is 0 .

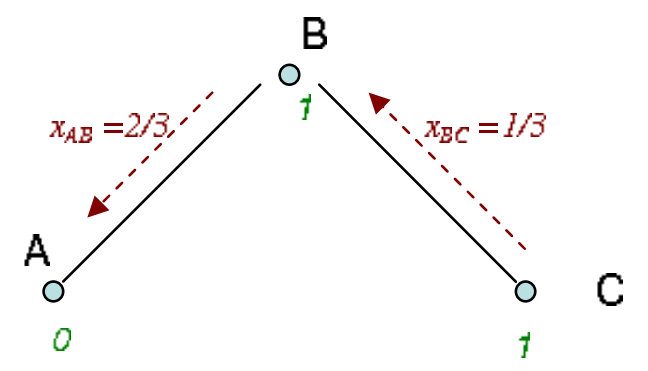

Figure 1. Bilateral Transfer Scheme

A bilateral (insurance) norm is a specification of consumptions for every linked pair of individuals, as a function of various observables. Such observables include individual identities $i$ and $j$ and, of course, the realizations $y_{i}, y_{j}, z_{i}$ and $z_{j}$. So a bilateral norm is just a function $b$ such that

$$
\left(c_{i}, c_{j}\right)=b\left(i, j, y_{i}, y_{j}, z_{i}, z_{j}\right)
$$

for every vector of realizations, subject to the constraint

$$
c_{i}+c_{j}=y_{i}+z_{i}+y_{j}+z_{j} .
$$

Some norms could be derived from bilateral welfare functions. Such a welfare function would depend on state-contingent consumptions $\mathbf{c}_{i}$ and $\mathbf{c}_{j}$ of the two agents, but could also depend on other variables, such as the ambient network component and the identity of the agents. Each pair — viewed as a social entity — by maximizing this function would generate a bilateral norm. For instance, if the bilateral welfare function consists of the sum of the utilities of the individuals, the resulting bilateral norm is equal sharing: the bilateral norm simply divides all available resources among the linked pair.

But bilateral norms also include a large class of sharing rules which are not easily amenable to a welfarist interpretation. For instance, suppose that each individual $i$ has full, unqualified access to some fraction $\alpha_{i}$ of her income, and must only share the rest of her resources using, say, an even split. The resulting transfer norm would then look like this:

$$
\begin{aligned}
c_{i} & =\alpha_{i} y_{i}+\frac{1}{2}\left[\sum_{k=i, j}\left(1-\alpha_{k}\right) y_{k}+z_{k}\right] \\
c_{j} & =\alpha_{j} y_{j}+\frac{1}{2}\left[\sum_{k=i, j}\left(1-\alpha_{k}\right) y_{k}+z_{k}\right]
\end{aligned}
$$

When $\alpha_{i}>0$ for all $i$, we call these, norms with a private domain.

A bilateral norm aggregates third-party obligations if the consumption of each individual depends on $z_{i}$ and $z_{j}$ through their sum $z_{i}+z_{j}$ alone and is continuously increasing in this variable.

Bilateral norms that aggregate third-party obligations can still be asymmetric (i.e., depend on the index $i$ and $j$ ), and they can also prescribe consumptions that are dependent on individual incomes in a variety of ways. Naturally, there is a large class of norms that satisfy this restriction. For instance, the equal sharing norm and the norms with a private domain do aggregate thirdparty obligations. 
Notice that a bilateral norm equivalently prescribes bilateral transfers for every linked pair and every realization.

2.4. Consistent Consumption Allocations. Recall that third-party transfers are endogenous functions of the state. Put another way, the society-wide operation of a bilateral norm not only generates transfers across every linked pair; it also generates the third-party receipts or debts that the pair takes as "given". Cutting through the implied circularity, a bilateral norm will yield a consumption allocation $\mathbf{c}(\theta)$ for everyone in the network, as a function of the realized state $\theta$. Call this a consumption allocation consistent with the norm, or a consistent consumption allocation for short. Obviously, with all the interpersonal interactions in the network, there may be more than one consumption allocation consistent with any given bilateral norm. The following proposition describes when this cannot happen, so that a unique prediction is obtained.

Proposition 1. Suppose that a bilateral norm aggregates third-party obligations. Then there is at most one consumption allocation consistent with that norm.

Proof. Suppose the assertion is false. Then there are two consistent allocations $-\mathbf{c}(\theta)$ and $\mathbf{c}^{\prime}(\theta)$ - and a state $\theta$ such that the induced vectors of consumptions across individuals in that state are distinct. Then there must be some linked pair $i j$ such that $c_{i}(\theta) \leq c_{i}^{\prime}(\theta)$ and $c_{j}(\theta)>c_{j}^{\prime}(\theta)$. But then at least one consumption is not strictly increasing in the sum of thirdparty obligations, a contradiction.

Recall the equal-sharing norm, in which every bilateral transfer is chosen to equalize consumption across a linked pair. It is easy to see that there is a unique consistent scheme associated with the equal-sharing norm, which entails "global" equal sharing of total output in any connected component of the network.

The equal-sharing norm, apart from its intrinsic interest, has the feature that there is some "multilateral norm" with which it is consistent; in this case, multilateral equal sharing. Bilateral transfer norms that allocate to each person a weighted share of consumption (depending perhaps on that person's identity or her income realization) also have this feature provided that the relative weights for every pair $\{i j\}$ equal the relative weights arrived at "indirectly" by compounding relative weights along any other path joining $i$ to $j$. It is also possible to generate bilateral schemes by maximizing welfare functions that depend (for every linked pair $i j$ ) on $\mathbf{c}_{i}$ and $\mathbf{c}_{j}$. Such functions may also depend on the ambient network component and the identity of the agents, as also their income realizations, particularly if these serve as proxies for "outside options".

It is also easy to find the consistent consumption allocation associated with any norm with a private domain. For a given income realization, any individual $i$ in a component $d$ of size $n$ will consume

$$
c_{i}=\alpha_{i} y_{i}+\frac{1}{n} \sum_{j \in d}\left(1-\alpha_{j}\right) y_{j}
$$

As a particular instance, take the norm in which all individuals keep half their income and share the remainder of their resources $\left(\alpha_{i}=\frac{1}{2}\right.$ for all $\left.i\right)$. Consider a three person network in which $B$ is linked to both $A$ and $C$. Figure 2 illustrates the transfers associated with the consistent allocation $(1 / 3,2 / 3,2 / 3)$ in a state in which B and C's income is 1 while A's income is 0 .

Now what about existence? Unfortunately, bilateral norms could be "incompatible enough" so that a consistent consumption allocation associated with that norm simply fails to exist. As an example, suppose there are three agents connected to each other in a circle. Assume that players 1 and 2 have a social norm that involves giving player 2 two-thirds of their joint endowment. Likewise, players 2 and 3 wish to give player 3 two-thirds of their joint endowment, 


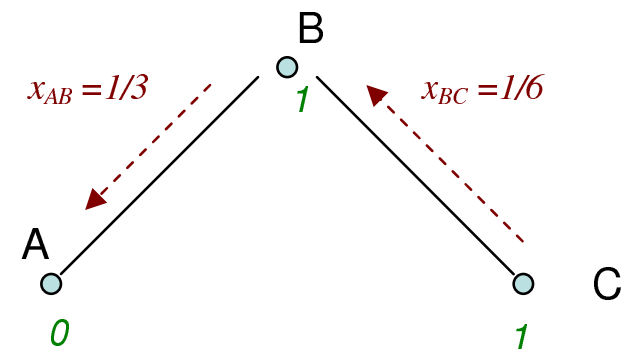

Figure 2. Consistent Allocation.

and a symmetric circle is completed by players 3 and 1 . Obviously, there is an incompatibility here, and it manifests itself in the nonexistence of a consistent consumption allocation. ${ }^{6}$

One way of seeing this incompatibility is to "follow" a natural fixed point mapping that would generate a consistent consumption allocation, were one to exist. If no consistent consumption allocation were to exist, such a mapping would prescribe larger and larger transfers as "best responses" in an ever-increasing spiral. If one proscribes unbounded transfers by assumption, the problem goes away. We record this as a proposition, for it tells us that there are no other existence problems except for the one just described.

Proposition 2. Suppose that for every linked pair ij, the bilateral transfer norm is continuous in $\mathbf{z}_{i}$ and $\mathbf{z}_{j}$, and that, in any state, the prescribed transfers cannot exceed some exogenous upper bound (say, the total output produced in society in that state). Suppose, moreover, that the norm never prescribes positive transfers from an individual with non-positive consumption to another with positive consumption.

Then a consistent consumption allocation exists, and exhibits positive consumption for every individual at every state.

We relegate the proof to the appendix.

Notice that every consistent consumption allocation $\mathbf{c}$ also implies an associated transfer scheme $\mathbf{x}$ : a collection of payments $x_{i j}(\theta)$ from $j$ to $i$ (positive or negative) for every linked pair $i j$ and every state $\theta$. The third party obligations are then defined by $z_{i}(\theta)=\sum_{k \neq j: i k \in g} x_{i k}(\theta)$, $z_{j}(\theta)=\sum_{k \neq i: j k \in g} x_{j k}(\theta)$. Observe that there may be several transfer schemes associated with the same consumption scheme: the uniqueness result of Proposition 1 does not apply to transfers.

We complete this section with a discussion of monotone norms.

2.5. Monotone Norms. Say that a bilateral norm is "monotone" if whenever more individuals are brought into a connected network by being connected to any particular individual, that individual's payoff increases. Intuitively, more individuals create better insurance possibilities, and a monotone norm should give some of the extra benefits to the individual serving as a "bridge".

Formally, suppose that $g$ and $g^{\prime}$, with $g \subseteq g^{\prime}$, are two connected components such that $N(g) \subset$ $N\left(g^{\prime}\right)$. Suppose, moreover, that $j k \in g^{\prime}$ only if $j k \in g$ for all $j, k \neq i$. Then, say that a norm is monotone if for every pair of associated consistent consumption allocations, one for $g$ and one for $g^{\prime}$, the expected payoff to $i$ under $g^{\prime}$ is higher than that under $g$.

\footnotetext{
${ }^{6}$ The incompatibility does not arise from the weights alone: the linkage structure matters as well. For instance, there is no existence problem if the three players are connected "in a line".
} 
Notice that monotonicity embodies more than a purely normative definition; it requires that a consistent solution not "misbehave" as we move across networks. ${ }^{7}$

It is easily seen that when agents are symmetric the equal division norm is monotone. It would be interesting to describe the full class of norms which satisfies monotonicity, though we do not know the answer to this question.

\section{Enforcement Constraints and Stability}

While a bilateral norm, as defined by us, comes from a fairly general class, it is time to emphasize a particular feature (already discussed in the Introduction). These norms are largely "normative" in that they take little or no account of self-enforcement constraints. But this isn't to say that such constraints do not exist. Each individual may recognize that as a social being she is constrained to abide by the transfer norm in her dealings with $j$, provided that she wants to maintain those dealings. But she may not want to maintain them. It may be that (in some states) the transfer she is called upon to make outweighs the future benefits of maintaining a relationship with $j$ under the bilateral norm. If that is the case, something must give, either the norm or the $i j$ link. Our paper takes the point of view that the norm is more durable than the link, and that the link will ultimately fail. ${ }^{8}$

In a network setting, an agent could choose to renege on some (or all) transfers that she is required to make under a particular bilateral norm. In line with the bulk of the literature on risk-sharing without commitment (see, e.g., Coate and Ravallion (1993), Fafchamps (1996), Ligon, Thomas and Worrall (2001) and Genicot and Ray $(2003,2005)$ ), individuals who are the direct victims of a deviation are presumed to impose sanctions on the deviant thereafter by not interacting with them.

This much may be clear, but nevertheless the extent of the punishment imposed on a deviant remains ambiguous. What about the rest of society, who were not directly harmed by the deviant? Do they, too, sever links with the deviant?

The answer to this question depends in part on what we are willing to assume about the extent of information flow in the society. In turn, this forces us to ask the question of just what the network links precisely mean. They certainly limit physical transfers, but do they also limit the flow of information? One possible interpretation is that the network represents a set of physical conduits and physical conduits alone, while information flows freely across all participants and is not constrained in any way by the architecture of the network. In this case the following notion of a punishment may be appropriate:

Strong Punishment. Following a deviation, every agent severs its direct link (if any) with the deviant, so that the deviant is thereafter left in autarky.

In models of informal insurance in groups with self-enforcement constraints, this is the commonly adopted punishment structure. But in such scenarios, there are no networks, insurance is

\footnotetext{
${ }^{7}$ One possible source of "misbehavior" is nonuniqueness of consistent schemes for a given network. While this in itself is by no means inconsistent with monotonicity, it makes the concept less intuitive.

${ }^{8}$ Generally speaking, should we conceive of norms as restricted or unrestricted by incentives? This is an important open question that we do not pretend to address in any satisfactory way. Norms may range all the way from the fully idealistic (purely derived from ethical considerations, such as equal-sharing) to the purely pragmatic (wary of all enforcement and participation constraints, with ethical matters only invoked subject to the limits posed by such constraints). In this paper, we take the point of view that norms are not constrained by incentives, but of course we do use such incentive constraints to see if the resulting bilateral norms will or will not survive.
} 
fully multilateral, and the event of a deviation is common knowledge among the group as a whole. In a situation in which network effects are under explicit consideration, the opposite presumption may seem more natural:

Weak Punishment. Following a deviation, only those agents who have been directly mistreated by the deviant sever their links (with the deviant).

In our view, this concept is more appropriate to the case at hand than strong punishment. In the model that we study, insurance is bilateral, and linked agent pairs know relatively little about the particulars of other dealings (only the aggregate of transfers made to or received from third parties). ${ }^{9}$ So it is entirely consistent to impose the restriction that while directly injured parties react, other agents do not, while strong punishments are more appropriate to a multilateral situation in which there are no restrictions on information flows and no network effects.

At the same time, if we take the network structure seriously, not just as a routeway for physical transfers but also for the flow of information, then we can define "intermediate" layers of punishment that are worth investigation in their own right. For instance, if I am an injured party and can communicate with those I am directly linked to, I can tell them about my experience. One might then adopt the equilibrium selection rule that all the individuals I talk to sever direct links (if any) with the deviant.

To be sure, once this door is opened, we might entertain notions in which the news of an individual's mistreatment "radiates outwards" over paths of length that exceed a single link, and all those who hear about the news breaks off direct links (if any) with the original deviant. There are many ways to model such a scenario: we take the simplest route by indexing such punishments by the length of the required path.

Level- $q$ Punishments. Following a deviation, all agents who are connected to a victim by a path not exceeding length $q$ (but not via the deviant) sever direct links (if any) with the deviant.

Figure 3 illustrates this punishment scheme for $q=1$. If in a given period individual 1 reneges on the transfers he owes to 4 and 5 , then not only 3 and 4 but also 5 will sever their links to 1 . This results in the subgraph $g^{\prime}$.

g
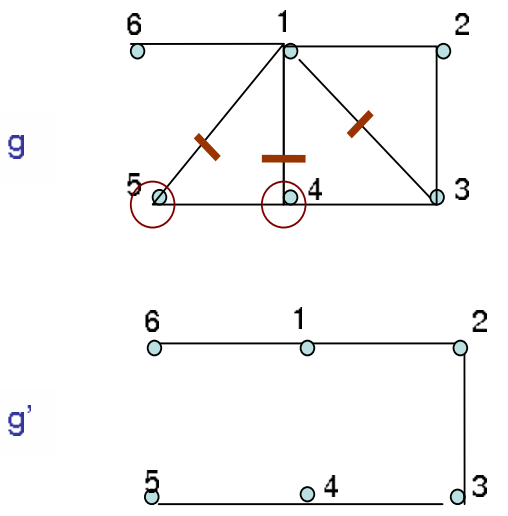

Figure 3. LeVEl- $q$ Punishment.

\footnotetext{
${ }^{9}$ However, for our model to have proper game-theoretic underpinnings — the formalities of which we do not explore here - it will be necessary to assume that the network itself is commonly known.
} 
In this definition, $q$ is to be viewed as a nonnegative integer, so that weak punishment may be thought of as a special case in which $q=0$. In this sense, level- $q$ punishments are quite general. ${ }^{10}$ In Section 6.2, we discuss some other punishment structure.

Notice that we effectively treat $q$ as an exogenous parameter to measure the extent of information flow. The reader may ask whether the passing-on of news about deviations may be detrimental to one's own interests (see a recent paper by Lippert and Spagnolo (2005) — though not on insurance - for more discussion on this point). This is an important question, but the issue is not critical here as our implicit punishment scheme is sustainable as a Bayesian equilibrium in a game in which network members send messages along their communication network (determined $q$ ) to report whether the transfer was made or not. No individual will make a positive transfer to another if they do not expect reciprocity. It follows that if a deviant believes that one of his neighbors has been informed of his misbehavior, he will stop making transfers to her anyway. Given these beliefs, it is an equilibrium strategy for individuals along the path from the victim to the neighbor to transmit the information on the deviation. Of course, there may be other equilibria in which badly treated partners resume dealings with the deviant or fail to pass the information, but we focus on the worst available punishments. Individuals who are direct victims or are informed of a deviation are presumed to transmit the information and impose sanctions according to the punishment scheme.

Now, given a level- $q$ punishment structure in place and given a norm, we may define $q$-stable networks. (Sometimes, when there is no danger of ambiguity, we shall simply use the term "stable" instead of $q$-stable.) Consider a community of $n$ individuals and a bilateral norm defined over all possible pairs of individuals. Because deviations result in the severance of links, we develop a recursive notion of stability. To this end, we begin with the empty graph in which all individuals live in isolation. The expected lifetime utility of an agent living in autarchy (normalized by the discount factor to a per-period equivalent) is

$$
v_{i}^{*}(\emptyset) \equiv \sum_{\theta} p(\theta) u\left(y_{i}(\theta)\right)
$$

The empty graph is obviously stable, as no transfers are called for and there is no question of a deviation. And there is only one payoff vector associated with that stable graph: $\mathbf{v}^{*}(\emptyset) \equiv\left\{v_{i}^{*}(\emptyset)\right\}$.

Proceeding recursively, consider a network $g$ representing the links among our $n$ individuals. Suppose that the set of stable subnetworks of $g$ has been defined, along with collections of stable payoff vectors for each of those stable subnetworks. Now consider the network $g$, and pick a consistent consumption allocation $\mathbf{c}$ with expected payoff vector $\mathbf{v}$. Fix a transfer scheme $\mathbf{x}$ associated with it. Consider any individual $i$. For any realization $\theta$, by abiding with the norm, $i$ obtains a lifetime (normalized) expected payoff of

$$
(1-\delta) u\left(c_{i}(\theta)\right)+\delta v_{i}
$$

In contrast, if $i$ deviates by not honoring commitments to a set of neighbors $S$, a level- $q$ punishment will set the new graph to $g^{\prime}$, which is obtained by removing from $g$ all direct links to $i$ that are from individuals who are no more than $q$ steps distant from some member of $S$ (but the connecting path should include the original deviant). Thus, the continuation payoff will depend on two things: the set of players who are her "victims", and the value of $q$ that determines the

\footnotetext{
${ }^{10}$ Of course, one might conceive of still more general punishment structures in which verifiable information decays - perhaps probabilistically — as it radiates along a path, but we avoid these for the sake of simplicity.
} 
punishment level. Formally, the payoff to $i$ of such a deviation is

$$
(1-\delta) u\left(y_{i}(\theta)+\sum_{j \notin S} x_{i j}(\theta)\right)+\delta v_{i}^{\prime} .
$$

How is $v_{i}^{\prime}$ in (2) determined? To describe the continuation payoffs following a deviation, we must adopt a convention that tells us the payoffs that accrue to player $i$ when she finds herself at some network $g^{\prime} \subset g$.

If $g^{\prime}$ is stable, it is to be expected that $i$ will enjoy a payoff of $v_{i}^{\prime}$, where this is the $i$ th component of some stable payoff vector for $g^{\prime}$. If $g^{\prime}$ is not stable, the resulting payoff will be presumably drawn from some stable subnetwork of $g^{\prime}$ itself. Two often-used devices to pin down the precise outcome in the face of potential multiplicity are "optimistic" and "pessimistic" beliefs (see, e.g., Greenberg (1990)). ${ }^{11}$ We assume that if $g^{\prime}$ is not stable then a subnetwork $g^{\prime \prime}$ would form where $g^{\prime \prime}$ is the or one of the largest stable subnetworks of $g^{\prime}$ to which $i$ belongs. In this case, $v_{i}^{\prime}$ is the $i$ th component of some stable payoff vector for $g^{\prime \prime}$. We do not insist on any particular selection rule at this conceptual stage, but we must take note of the "baseline" graph that player $i$ induces on her deviation. This depends on two things: the set of players who are her "victims", and the value of $q$ that determines the punishment level.

Furthermore, notice that our formulation of the payoff following a deviation implicitly assumes that an agent's deviation does not affect the ability of other agents to fulfill their commitments. In other words, we suppose that all other agents continue to conform to the norm after the deviation of agent $i$. This restriction stands in contrast to the assumption made in the literature on systemic risks and interbank credit (Allen and Gale (2000), Rochet and Tirole (1996) and Freixas, Parigi and Rochet (2000)). In models of interbank lending, the liquidation of assets held by bank $i$ in bank $j$ affects bank $j$ 's ability to honor its commitment, and can result in financial contagion, where the failure of one bank triggers the failure of other banks in the network. We abstract from this important but difficult issue in this paper.

Comparing (2) and (1), we may therefore say that a network $g$ is $q$-stable under a given bilateral norm if it has a consistent consumption allocation (with expected payoff vector $\mathbf{v}$ ) and associated transfer scheme such that for every player $i$, every state $\theta$ and every set of direct neighbors $S$ of $i$,

$$
(1-\delta) u\left(y_{i}(\theta)+\sum_{j \notin S} x_{i j}(\theta)\right)+\delta v_{i}^{\prime} \leq(1-\delta) u\left(c_{i}(\theta)\right)+\delta v_{i} .
$$

Stability requires that for all possible state realizations, the stipulated transfers be self-enforcing.

If $q$ is small, then punishments are "weak": not many individuals punish the miscreants. Indeed, for $q=0$ we obtain precisely the notion of weak punishment introduced earlier. If $q$ is large we approximate strong punishment and indeed that is what we get if the network is connected to begin with. In any case, "strong stability" can always be defined by setting $v_{i}^{\prime}$ in (2) to $v_{i}^{*}(\emptyset)$; no recursion is needed.

Notice that $q$-stability exploits fully an awareness of repeated interaction between individuals and is therefore different from the stability concepts in Jackson $(2001,2004)$ and Baya and Goyal (2000).

\footnotetext{
${ }^{11}$ For instance, in the former case, $v_{i}^{\prime}$ would be the maximum value of $v_{i}$ drawn from all stable payoff vectors drawn from any (stable) subgraph of $g^{\prime}$.
} 
Inequality (3) can be rewritten as

$$
u\left(y_{i}(\theta)+\sum_{j \notin S} x_{i j}(\theta)\right)-u\left(y_{i}(\theta)+\sum_{j} x_{i j}(\theta)\right) \leq \frac{\delta}{1-\delta}\left[v_{i}-v_{i}^{\prime}\right] .
$$

This inequality requires that the short term deviation gain from not making the transfers be smaller than the long term gain from remaining in the original risk-sharing network. This inequality makes it clear that when $\delta$ is close to one, long-term effects - the gap between $v_{i}$ and $v_{i}^{\prime}$ - dominate while short-term effects - the size of the one-time deviation gain - matter for lower values of the discount factor.

We are interested in analyzing the role of the network architecture on network stability. To this purpose, we will isolate and analyze two different effects that pertain to social networks are critical for their stability: the transit effect, a short-term effect, and the punishment capacity of networks, a long term effect. The next section focuses on the latter aspect by looking at high values of the discount factor. In Section 5, we then consider lower discounting values to examine transit or "bottleneck" effects.

\section{Stable Networks for High Discount Factors}

In this section we characterize the set of $q$-stable networks for a given bilateral norm. The following notion of a "sparse connectedness", related to the length of minimal cycles connecting any three agents in a network, will be central to the analysis. ${ }^{12}$

4.1. Sparseness. For any triple of agents $(i, j, k)$ such that $(i, j)$ and $(j, k)$ are connected in the network, compute the length of the smallest cycle connecting $i, j$ and $k, \ell(i, j, k)$. By convention, if there is no cycle connecting those three points, we define $\ell(i, j, k)=2$. For any integer $q \geq 0$, say that a graph $g$ is $q$-sparse if all minimal cycles connecting three agents in the network have length smaller or equal to $q+2$.

Note that if a network is $q$-sparse, then it is $q^{\prime}$-sparse for all $q^{\prime} \geq q$. Observe also that all networks which only have trees as their components are 0 -sparse - there are no cycles connecting any three points in a tree - and that this fully describes the set of 0-sparse networks. On the other hand, the complete network is 1-sparse, as any three agents are connected by a cycle of length 3. Actually, we show in the appendix that a network is 1-sparse if and only if its components are clusters connected by bridge-nodes (see Observation 1 in the appendix). Finally, for a connected network of size $n$, the graph architecture with the highest index of sparseness is the circle, where any three agents are connected by a cycle of length $n$, so that the circle is $(n-2)$-sparse. As an illustration, Table 1 characterizes the lowest $q$-sparseness of the different network architectures for 8 connected agents illustrated in Figure 4.

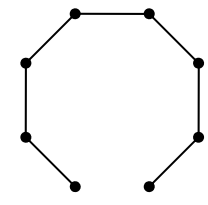

a.

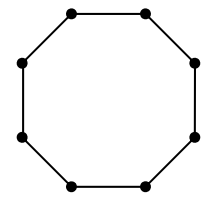

b.

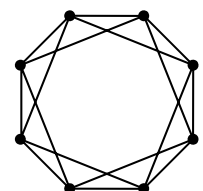

c.

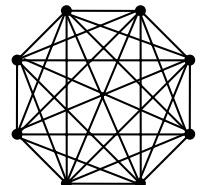

d.

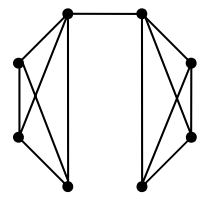

e.

FiguRE 4. $q$-SPARSENESS \& CYCLES

\footnotetext{
${ }^{12}$ We are grateful to Anna Bogomolnaia whose comments suggested this definition to us.
} 


\begin{tabular}{llc}
\hline & graph & minimal sparsity \\
\hline a. & tree & 0 \\
b. & circle & 6 \\
c. & 3 neighbors & 2 \\
d. & complete graph & 1 \\
e. a bridge & 1 \\
\hline
\end{tabular}

TABLE 1. SPARSENESS.

\subsection{Characterization of $q$-Stability.}

Proposition 3. Suppose that a bilateral norm is monotonic and aggregates third-party obligations. Then a network $g$ is q-stable if and only if it is $q$-sparse.

It is very important to note that $q$-sparseness is a purely graph-theoretic property. It requires no knowledge of utility functions, the set of possible income realizations, and the stochastic law that governs such realizations. In contrast, $q$-stability is a more complex game-theoretic notion. Not only do we need knowledge of utilities and endowments to define the concept, we need the usual repeated game apparatus of deviations and punishments. Therefore, despite the linguistic similarity in terminology, $q$-stability and $q$-sparseness are very distinct concepts, and indeed this is why our characterization is potentially useful.

Proof. The proof of necessity uses two steps. The first step shows that for the bilateral norms under review, no punishment can be imposed on a deviant unless it is severe enough to break down the connected component of which the deviant is part.

LEMma 1. Suppose that a bilateral norm aggregates third-party obligations. Then, assuming that a consistent consumption allocation exists for $\mathrm{g}$, exactly the same allocation is consistent for every connected subnetwork of $g$.

For a formal proof, see the appendix. Bilateral norms that aggregate third-party obligations manage to impose the same consumption structure over every set of agents, provided that they are connected. The particular structure of connectedness does not matter. For instance, the equal division bilateral norm actually gives rise to global equal division over any set of agents, as long as they form a connected network.

The second main step is a restatement of $q$-sparseness.

LEMma 2. A graph $g$ is $q$-sparse if and only if, for every linked pair ij $\in g$, the graph formed by removing from $g$ the links to $i$ along all paths of length $m \leq q+1$ between $i$ and $j$ has strictly more components than $g$.

Once again, the proof is postponed to the appendix. Now connect Lemmas 1 and 2. Consider a network and a bilateral norm that aggregates third-party obligations. Assume that an agent $i$ reneges on the transfers she owes to one or more partners. Then the immediate victims certainly sever their links to $i$, and so do all individuals (connected to $i$ ) who are connected to any of them (but not through $i$ !) via a path of length $q$ or less. If the graph $g$ is not $q$-sparse, then, in the resulting subgraph, $i$ will not find herself in a smaller component, by Lemma 2. By Lemma 1 , there can be no reduction in continuation utilities following the deviation, so $q$-stability is not possible. 


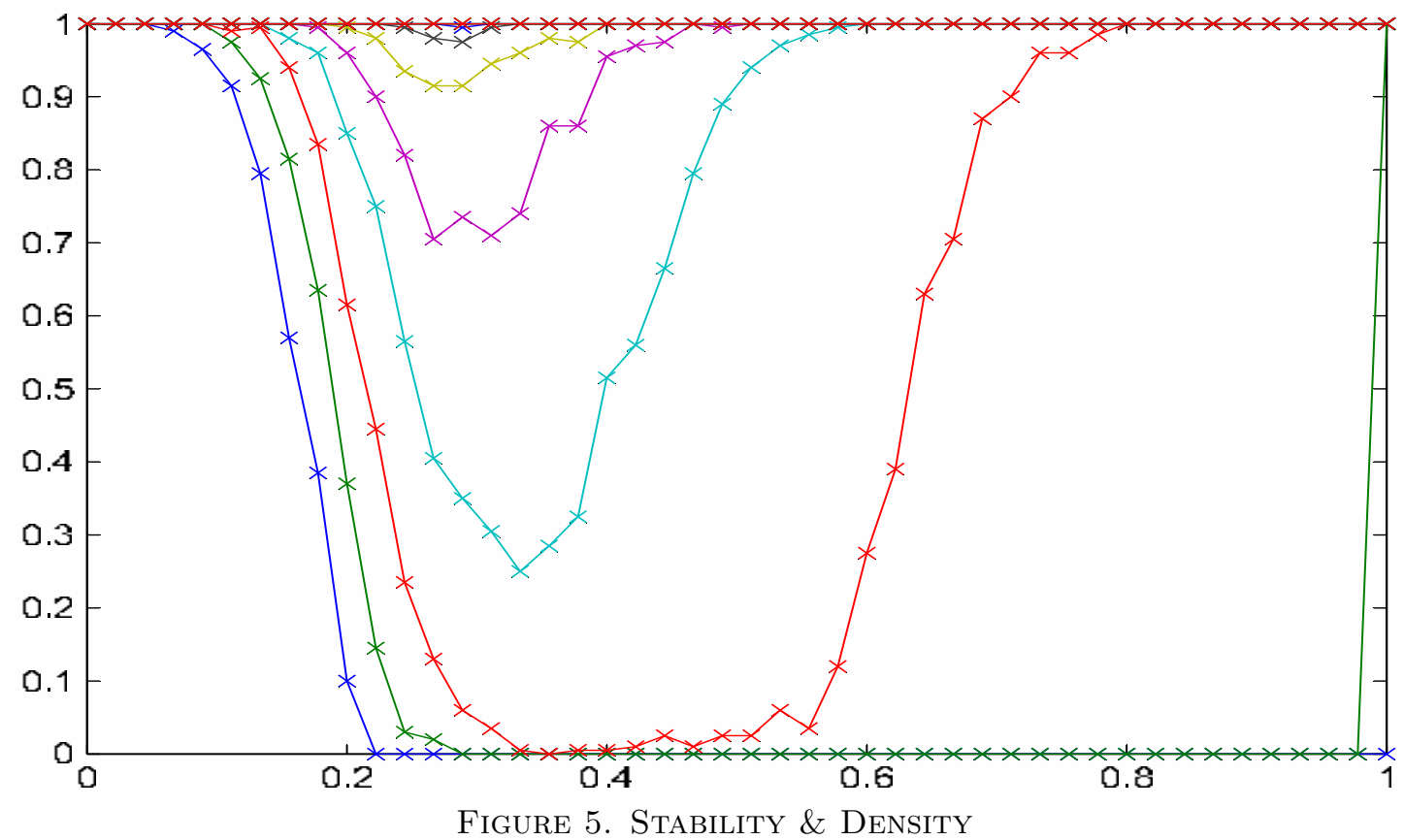

The sufficiency direction is trivial. For monotone norms, $q$-sparse networks must cause a discrete loss in expected utility (Lemma 2 again). This is enough to yield $q$-stability for all discount factors close enough to 1 .

4.3. Strong and Weak Punishment. It is particularly interesting to contrast the stability of networks across different punishment schemes. For values of $\delta$ close to 1 , every network is strongly stable. But this is no longer true as $q$ comes down, weakening the flow of information. The following corollary summarizes the two extremes.

COROLlary 1. Suppose that the bilateral norm is monotonic and aggregates third-party obligations. Then a network is stable under weak punishment for discount factors close to unity if and only if it has only trees as components. In contrast, under strong punishment, every network is stable for high enough discount factors.

4.4. Density and Stability. Figure 4 and Table 1 suggest that there is no monotonic relation between the sparseness of a network, and its density as measured by the number of links. Very thin networks such as trees and very dense networks such as the complete graph tend to have low sparseness indices. Intermediate graph structures on the other hand have higher sparseness indices, suggesting a U-shaped relation between sparseness and the number of links. For instance, for $q=1$, only the line $(a$.$) , the complete graph (d$.$) and the bridge (e$.$) architectures are stable.$

In order to test this hypothesis, we run simulations on the network structure. Consider a community of ten individuals. We generate random networks of size 10 and for each network compute its sparseness index and its density index (the number of links divided by the maximal number of links, $\frac{10 * 9}{2}=45$ ). Figure 5 maps the proportion of $q$-stable networks on network density. Each curves correspond to a different level of punishment $q$ ranging from 0 to 9 . Naturally, the stronger the punishment the larger the proportion of stable graphs, such that higher curves correspond to larger $q$ 's. As the analysis suggests, networks of intermediate density tend to be unstable. 
Consider a densely connected network. Our result suggests that if, following some exogenous shocks, some links are broken, it could destabilize the network and create an unravelling of the existing relationships, resulting in a sparsely connected graph.

4.5. Stability and Clustering Coefficient. For intermediate values of $q$, stability is related to the clustering coefficient defined in the social network literature on "small world" (see Wasserman and Faust (1994), and Watts and Strogatz (1998)). Recall that $\aleph_{i}$ is the neighbor set of $i$ (the set of individuals with direct links to $i$ ). The clustering coefficient for $i$ in $g$, given that $\left|\aleph_{i}\right| \geq 2$, is the actual number of links among $\aleph_{i}(i)$ divided by the maximum number of links among $i$ 's neighbor set $\left|\aleph_{i}\right| *\left|\left(\aleph_{i}-1\right)\right| / 2$. It measures the propensity for $i$ 's neighbors to be linked to each other. The clustering coefficient of a graph is then simply the average clustering coefficient of its members. Intuitively, for intermediate level of punishment, more clustered networks are better able to ostracize deviants and therefore are more likely to be stable.

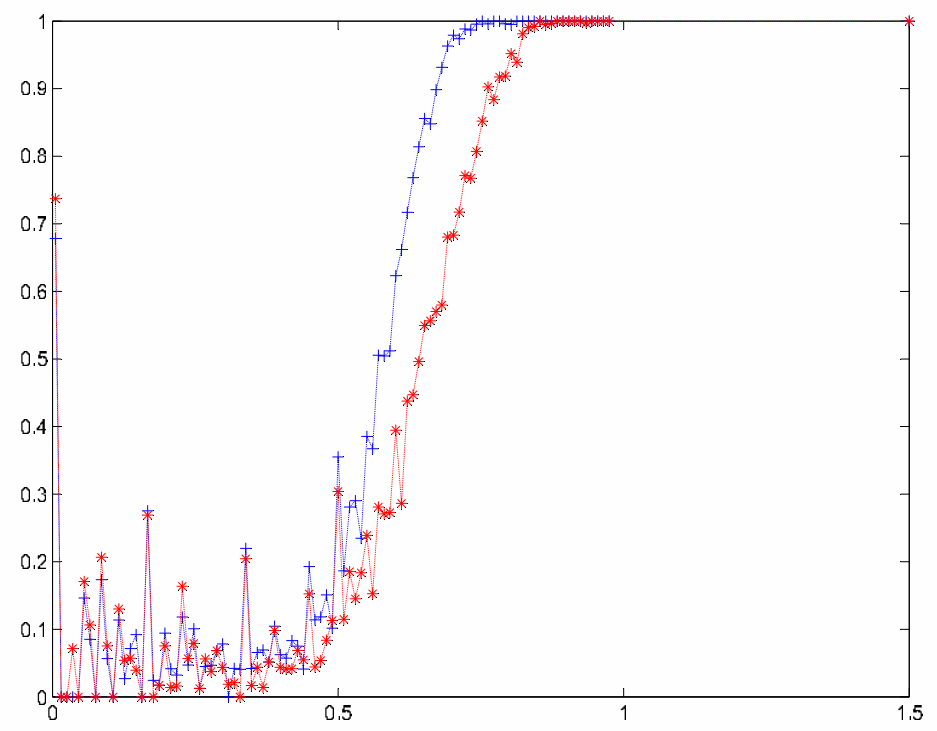

Figure 6. Stability \& Clustering

Using simulations, we can simulate a large number of networks of size 10 for each possible density level, and map their universal $q$-stability on their clustering coefficient. Figure 6 reports the results. The graph connecting the asterisks shows the proportion of universally $q$-stable networks for $q=2$ as a function of the clustering coefficient. [The graph connecting crosses will be used later.] Except for the empty graph, the relationship between stability and clustering is clearly positive.

Note that, in a different model, Vega-Redondo (2003) uses numerical simulations to examine the importance of the architecture of the network: its density and cohesiveness, to transmit information on deviant in repeated Prisoner's Dilemma games.

\section{Short-Term or Transit EFFECTS}

In this Section, we consider the effect of network architectures on stability for lower values of the discount factor. We concentrate on the short-run effects of networks - the relation between 
the architecture of the network and the instantaneous gain from reneging on the transfers. In order to isolate these short-run effects, we consider a specific model with equal sharing norm and strong punishment. In that model, the long run utility of an agent who complies to the insurance scheme, $v_{i}$, only depends on the size of the component and the long run utility following a deviation, $v_{i}^{\prime}=v_{i}(\emptyset)$, is independent of the network and of the set of links on which an agent deviates. Hence, the network architecture only affects the self-enforcement constraint through the short run effects of a deviation.

5.1. Bottleneck Agents. Under the equal sharing norm, for a fixed component $d$ of size $m$ and a state $\theta$ with income realization $\mathbf{y}$,

$$
y_{i}+\sum_{j} x_{i j}(\theta)=\sum_{i} y_{i} / m .
$$

The short run gain from a deviation at state $\theta$ is thus given by:

$$
u\left(y_{i}+\sum_{j \mid x_{i j}>0} x_{i j}(\theta)\right)-u\left(\sum_{j} y_{j} / m\right)
$$

In order to check the stability of a graph, we thus need to check, component by component, and for every vector of income realization, the incentives of the agent(s) for which (5) is maximal. We call this agent or these agents the bottleneck(s) of the transfer scheme. Intuitively, the bottleneck agent is an agent who receives and redistributes transfers and has the highest incentive to "take the money and run". If all agents face the same maximal short-term incentive to deviate (5) in at least one state, we say that there is no bottleneck agent.

For general graphs where transfers can flow along different links, the identification of bottleneck agents is a complex task, as it depends on the exact routing of transfers. The analysis of bottleneck agents is much easier in trees, where every pair of agents is connected by a single path. In order to gain intuition, we illustrate below the concept of bottleneck agents for two different trees: a star and a line with four agents.

Consider the simplest case in which the endowments are i.i.d. across agents and take on just two values: $h$ with probability $p$ and $\ell$ with probability $(1-p)$, with $h>\ell>0$. Let $\eta$ denote the number of high realizations. For values of $\eta \in\{1, \ldots 3\}$, Figure 7 and Figure 8 illustrate the state realization and underline the agent for which (5) is maximal in the star and the line.

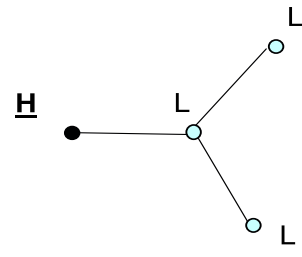

$\mathrm{k}=1$

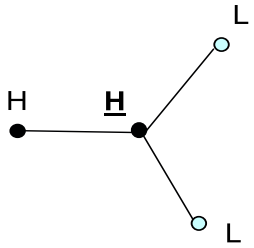

$\mathrm{k}=2$

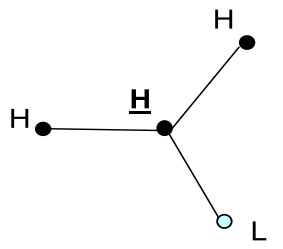

$\mathrm{k}=3$

Figure 7. Bottleneck Agent in the Star. 


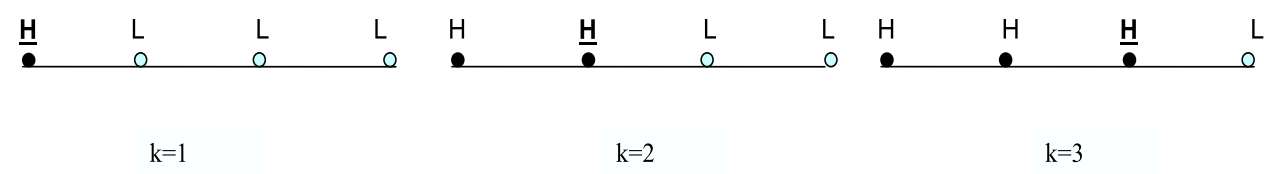

Figure 8. Bottleneck Agent in the Line.

For any number of good realizations, we can actually show that the constraint faced by the bottleneck agent is the same in the two graphs. ${ }^{13}$ For any $\eta$ good realizations, the bottleneck agent receives transfer from $\eta-1$ agents both in the star and the line, and her maximal deviation payoff is given by

in both graphs.

$$
h+\frac{(\eta-1)(n-\eta)(h-l)}{n}
$$

The next result shows that this is not an accident: both the star and the line belong to a special class of decomposable networks for which the short-run incentives to deviate are extremely high.

5.2. Decomposable Networks. We define a subnetwork of $g$ originating at $i$ as a subgraph $g^{\prime}$ obtained by removing only some of $i$ 's links : $g^{\prime} \subset g$ and if $j k \in g$ then $j k \in g^{\prime}$ for any $j, k \neq i$.

A node $i$ is said to be critical of degree $\gamma$ if there exists a subnetwork $g^{\prime}$ originating at $i$ such that, in $g^{\prime}, i$ belongs to a component of size $\gamma$;

A connected graph $g$ of size $m$ is decomposable if for any $\gamma=1, \ldots, m-1$, there exists a critical node $i$ of degree $\gamma$.

Intuitively, a connected network of size $m$ is decomposable if for any $\gamma \in[1, m-1]$, there exists a way to decompose the network into two subnetworks of size $\gamma$ and $m-\gamma+1$ such that a single agent (the critical agent) controls all transfers from agents in one subnetwork to agents in the other subnetwork. It is easy to identify the bottleneck agent in a decomposable network. For any realization of the state, one only needs to compute the value of the size $\gamma$ of the subnetwork for which the critical agent has the highest incentive to deviate. Both the star and the line are decomposable networks. In the star, the central individual is critical of degree $\gamma$ for all $\gamma$. In the line where agents are ordered as $1,2, \ldots, m$, the $\gamma$ th agent is critical of degree $\gamma$. The next Proposition shows that all decomposable networks have the same stability property and that they are the networks for which the short-run incentives to deviate are the highest.

Proposition 4. For any two decomposable connected networks $g$ and $g^{\prime}$ of size $m, g$ is stable if and only if $g^{\prime}$ is stable. Furthermore, if $g$ and $g^{\prime}$ are two networks of size $m, g$ is decomposable and $g^{\prime}$ is not, then $g^{\prime}$ is stable if $g$ is stable.

The intuition for this result is the following. Consider a connected and decomposable network. For any given income realization, call givers the individuals who are called upon making a positive net transfer to the network. Take a realization $\mathbf{y}$ and assume that there are $\gamma(\mathbf{y})$ givers. It follows from the decomposability of the network that there is a critical node $i$ of degree $\gamma$. Since the income distribution is symmetric, there is a permutation of $\mathbf{y}$, say $\mathbf{y}^{\prime}$ in which all givers are in the component of size $\gamma$ of the subnetwork originating at $i$. We show that individual $i$ 's incentive

\footnotetext{
${ }^{13}$ The fact that the two networks have the same short-run effects on stability does not imply that they are equivalent. Intuitively, insurance seems easier to sustain if agents form a line than a star. This is due to the fact that with weak punishments, the hub of the star will only lose the ability to insure with one agent if he deviates on one link, whereas an agent in line will typically break the community into groups of different sizes and lose the ability to insure with a larger number of agents.
} 
constraint is the hardest to satisfy under $\mathbf{y}^{\prime}$, and that this constraint is at least as difficult to satisfy than any of the constraints under the original income realization $\mathbf{y}$. Moreover, this particular constraint is the very same for all decomposable and connected networks of same size. Since this is true for all income realizations, all decomposable and connected networks of same size will have the very same stability properties. Furthermore, if a network is not decomposable, either there exists a critical agent of degree $\gamma$ for the realization for which the incentive constraint is hardest to satisfy or not. In the first case, the network has the same stability property as a decomposable network ; in the second case, the incentive constraints are harder to satisfy in the decomposable network.

This line of reasoning shows that the addition of links can only relax the bottleneck effect, as it allows to reroute transfers along new links. Hence we obtain the following corollary, which uses the same argument as Proposition 4

Corollary 2. Consider two graphs $g$ and $g^{\prime}$ with the same components and $g \subset g^{\prime}$. Then if $g$ is stable, $g^{\prime}$ is stable.

Remark that, as a consequence, for any connected graph $g$, if $g$ is stable, the complete graph $g^{c}$ is stable. Examples can easily be provided to show that the reverse implication does not necessarily hold.

Notice that this remark does not show that the complete graph is the easiest graph to sustain. The inequality is only true among connected graphs and disconnected graphs, with smaller components, may be easier to sustain. In fact, there is no obvious reason why the enforcement constraint faced by community members should be monotonic in the number of agents in a component. To illustrate this point consider the simple case, already used earlier, in which the endowments are i.i.d. across agents and take on just two values: $h$ with probability $p$ and $\ell$ with probability $(1-p)$, with $h>\ell>0$. The following example computes the threshold value $\delta$ as a function of the size of the graph. It appears that the threshold value is non monotonic in the size of the graph.

ExAmple 1. Take utilities to be $u(c)=2 c^{1 / 2}, \ell=0, h=1$ and $p=0.2$.

In a complete graph, no transfer is ever mediated. This implies that the binding constraint for an agent is whether to keep her income when it receives a high shock or not. In other words, the maximal deviation is given by

$$
u(h)-u\left(\frac{\eta h+(n-\eta) l}{n}\right)
$$

This expression is clearly maximized when $\eta=1$ so the constraint becomes

$$
(1-\delta) u(h)+\delta v^{*}(1) \leq(1-\delta) u\left(\frac{h+(n-1) l}{n}\right)+\delta v^{*}(n)
$$

where

$$
v^{*}(n)=\sum_{\eta=0}^{n}\left(\begin{array}{c}
n \\
\eta
\end{array}\right) p^{\eta}(1-p)^{n-\eta} u\left(\frac{\eta h+(n-\eta) l}{n}\right)
$$

We compute

$$
\delta(n) \equiv \frac{1-(1 / n)^{1 / 2}}{1-(1 / n)^{1 / 2}-0.2+\sum_{\eta=0}^{n}\left(\begin{array}{c}
n \\
\eta
\end{array}\right) 0.2^{\eta} 0.8^{n-\eta}(\eta / n)^{1 / 2}}
$$

Figure 9 pictures $\delta$ as a function of $n$ for $n \in\{1, \ldots, 100\}$. 


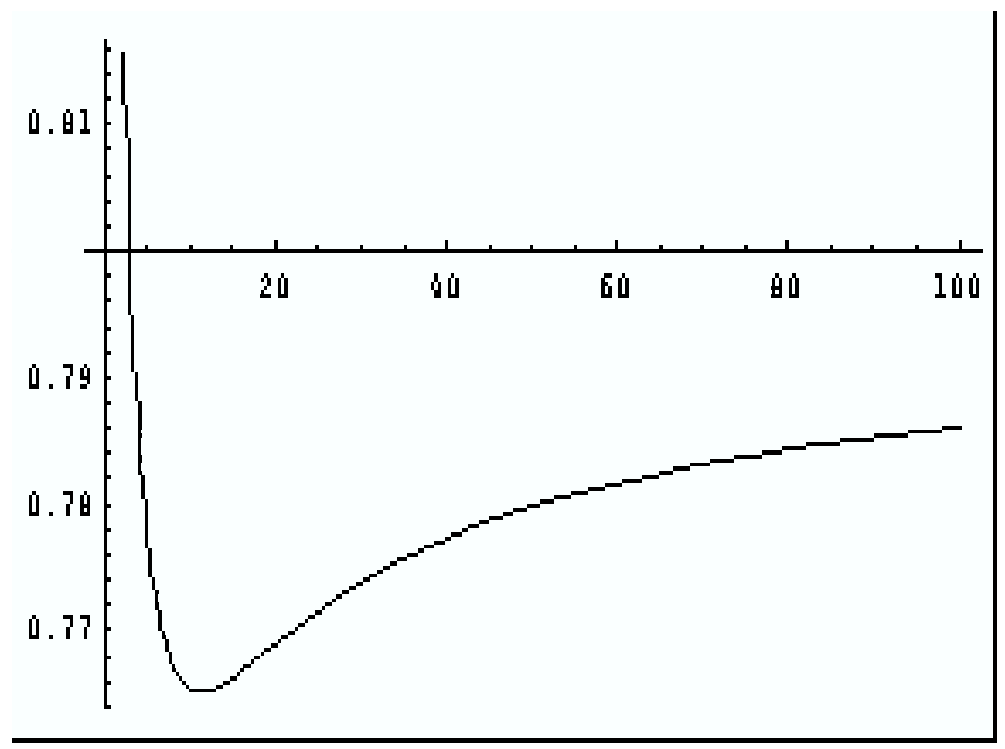

Figure 9. An Illustration of Example 1.

5.3. Mixing Long Run and Short Run Effects. In general, the stability of networks depends on the interplay between long run and short run effects. For different values of the discount factor, different stable networks will emerge, as the short run effect dominates for low values of $\delta$ and the long run effect for high values of $\delta$. In order to gain intuition about the variation of stable networks with changes in the discount factor, we consider here a simple example of an equal sharing norm with weak punishment.

EXAMPLE 2. Endowments are i.i.d. across agents and take on just two values: $h$ with probability $p$ and $\ell$ with probability $(1-p)$, with $h>\ell>0$. The following parameters are set through the example: $\ell=0$ and $h=1$, and individuals are assumed to have utilities $u(c)=2 c^{1 / 2}$.

Figure 10 depicts all stable networks for three agents, for two different values of $p$, as a function of t the discount factor. In the upper panel, $p=0.2$ and $p=0.5$ in the lower panel.

Because we consider weak punishment (or 0-stability), trees emerge as the unique stable networks for high values of the discount factor. For lower values of $\delta$, complete graphs emerge as stable network architectures. However, stable networks are not necessarily connected. Surprisingly, for $p=0.2$, the complete connected graph is stable for low values of $\delta$, then becomes unstable, and becomes stable again for higher values of the discount factor.

\section{Discussion and Possible Extensions}

6.1. Sparseness and Decomposability. The analysis of long run and short run effects has led to the definition of two properties of social networks: sparseness and decomposability. At a superficial level, these two properties seem to be connected, as they both imply that the graph possesses a small number of links. However, because they reflect very different properties of the network, it is easy to see that sparseness and decomposability are different concepts, and that sparse graphs may not be decomposable, and decomposable graphs may not be sparse. Figure 


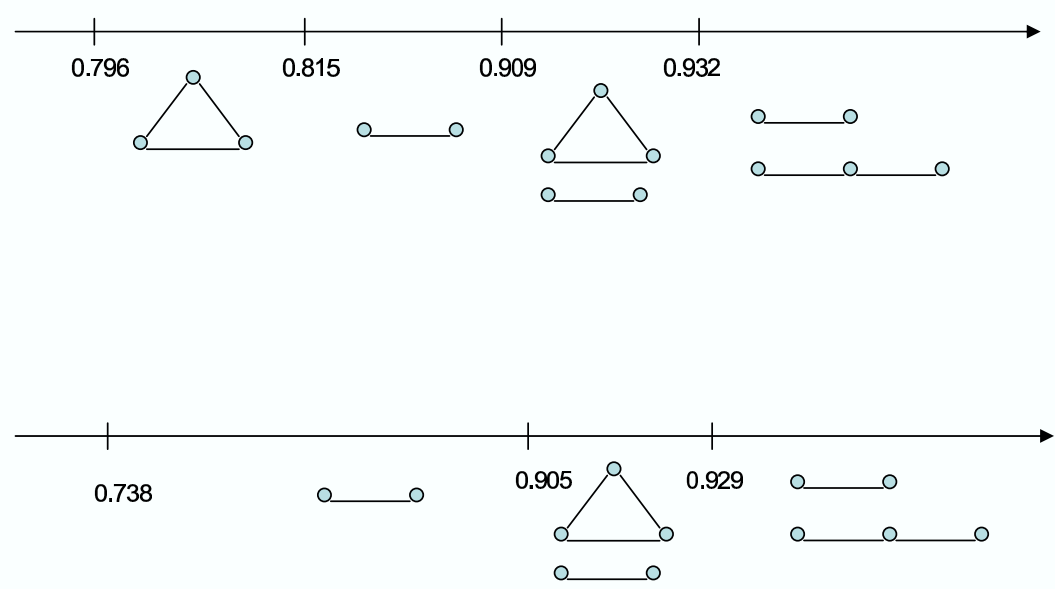

Figure 10. An Illustration of Example 2.

11 illustrates this very clearly with two networks of 7 agents. The first graph shows a tree recall that trees have sparseness 0 - which is not decomposable, while the second network is decomposable and has sparseness 2 .
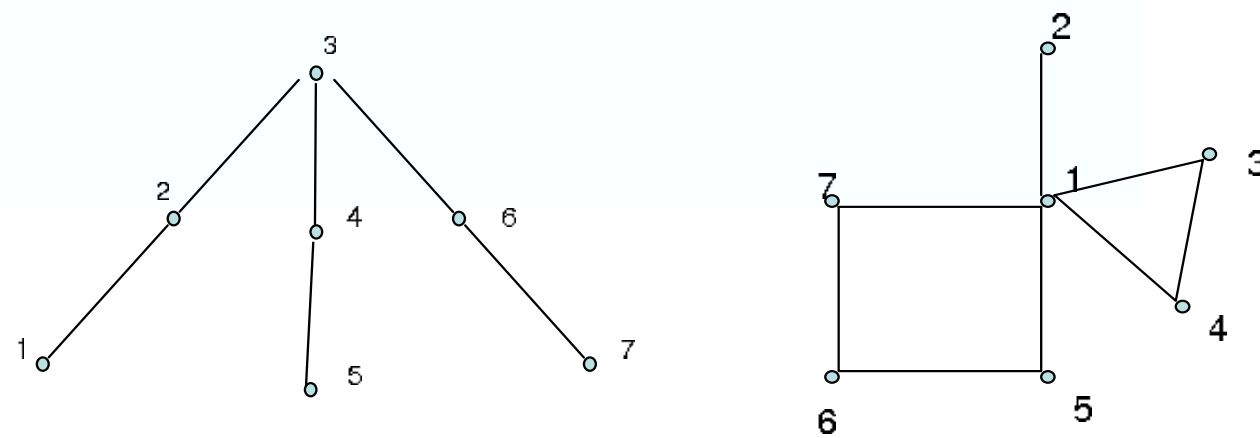

Figure 11. Decomposability and Sparseness.

6.2. Communication Protocol and other Punishment Schemes. Our $q$-level punishment captures two important features of communication within networks: it is generally limited and it depends on the graph itself.

One could imagine pushing these ideas further and have a punishment consistent the following communication protocol: information is transmitted only via individuals who know the person concerned. The idea is that before relaying to a person information on $x$, one would first ask to the person if she knows $x$. If she does, then the information is transmitted but otherwise not. In our model, knowing the person is taken to mean having a link with the person. We can define a new punishment structure using this particular communication protocol. 
InFORMATIOn Sharing. Information on a deviation is transmitted from an agent to another if and only if they are linked to each other and to the deviant. Following a deviation, all informed agents sever direct links with the deviant.

Formally, say that $i$ is strongly connected to $k$ via $j$ if there exists a sequence of individuals $\left\{i^{0}=j, i^{1}, \ldots i^{n}\right\}$ such that $i^{m} i^{m+1} \in g$ for all $m=\{1, \ldots n-1\}$ and $i^{m k} \in g$ for all $m=1, \ldots n$.

Using arguments similar to Proposition 3, it is is easy to show that for monotone norms and a discount factor close to 1 , a network $g$ is stable under information sharing if and only if for every linked pair $i j \in g$, the graph formed by removing from $g$ the links between $i$ and all individuals strongly connected to $i$ via $j$ has strictly more components than $g$.

Intuitively the clustering coefficient discussed in Section 4.5 will be particularly important for stability under our information protocol. We illustrate this using a specific example and simulations. Figure 12 pictures three different graphs with 8 individuals having 3 links each. The clustering coefficient of each individual is indicated next to her node. In the first network, the wheel, each individual has a clustering coefficient of 0 . It follows that, for high values of $\delta$, this graph won't be stable. Next, consider the neighbor network. If individual $i$ in the Figure defects on a transfer to individual $j$, where both have clustering coefficients of $2 / 3$, then our information protocol would isolate and effectively punish $i$. In contrast, if it is individual $k$ (whose clustering coefficient is only $1 / 3$ ) who defects on $i$, we see that our information protocol will be unable to punish him. Hence, graph $b$. is also unstable. Finally, look at the cliques. Here individual are clustered in two cliques of four individuals. Their clustering coefficient is 1 and therefore it is possible to fully isolate any deviant. Hence, the graph is stable for monotone norms.
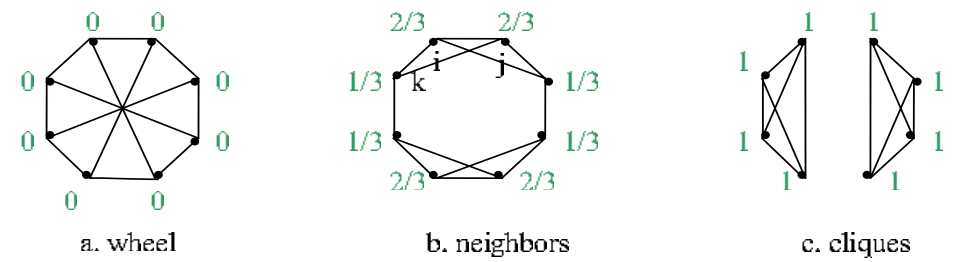

FiguRE 12.

Using the same simulation technique as in Section 4.5, we simulate a large number of network of size 10 for each possible density level and assess their their stability under our information protocol. Figure 6 reports the results. The crosses represent the proportion of universally stable graph under our information protocol for different clustering coefficients. Except for the empty graph, we see that the relationship between stability and clustering is clearly positive under our information protocol, and even stronger than for $q$-punishment.

\section{Conclusion}

In this paper, we develop a model of risk-sharing in social networks. Only individuals who are linked can make transfer to each other. There are two important features to our model. First, a risk-sharing arrangement at the level of network results from a collection of bilateral arrangement among linked individuals. Second, for each linked pair of individuals, bilateral insurance norms prescribes consumptions and transfers as a function of the individuals' income, identities and their net obligations to others in the network. As these obligations are endogenous, we focus on consumption allocation and transfer schemes that satisfy a society-wide consistency: a consistent transfer scheme is a fixed point of these bilateral state-contingent transfers. 
After characterizing conditions for existence of consistent consumption allocation schemes, we assess the stability of insurance networks stressing that to be stable a risk-sharing network must be self-enforcing. Two important factors affect the stability of insurance networks: a transit effect and an information effect determining the severity of punishment in the network.

Information effects can be isolated by looking at discount factor close to one. We show that for monotone norms the stability of a network can be fully characterized by a measure of its sparseness. What is remarkable is that the sparseness of a network is purely a function of its architecture, while its stability depends on individuals having incentive to make the prescribed transfers. This result implies that, for discount factor close to unity, all networks are stable under strong punishment, while only networks of trees are stable under weak punishment. For intermediate level of punishment both very dense graphs and minimally connected graphs are stable while graphs of intermediary density tend to be unstable.

For lower discount factors, the architecture of the network is also important for the transit effect. A high amount of transfers going through a particular agent provides her with a strong short term incentive to deviate from the insurance scheme. Such "bottleneck effect" can be clearly illustrated for the equal sharing norm in the case of strong punishment. We define a class of decomposable networks - a concept that is again purely descriptive of their architecture. We are then able to show that all decomposable networks share the same stability conditions, and have the most acute transit effect. In other words, they are the hardest networks to sustain.

By introducing a model of risk-sharing networks, this paper opens the door to much future research. There are a number of possible extensions of our analysis. First, different social norms have different stability properties. Comparing them would give us insights on the endogenous emergence of insurance norms. Second, we have explored one source of instability of insurance networks: the lack of commitment. There are many other possible sources of instability. For instance, many other interesting issues emerge in situations where incomes are only imperfectly observed. Finally, allowing for link formation and analyzing the endogenous formation of risksharing networks is an important issue for future work.

\section{Proofs}

8.1. Proof of Proposition 2. [Existence]. Fix a network $g$. Denote by $M(\theta)$ the size of the bound on transfers in state $\theta$ Let $\mathbf{X}$ be a Euclidean cube, where $\mathbf{x} \in \mathbf{X}$ is a vector of transfers $x_{i j}$, one for every linked pair, such that $x_{i j} \in\left[-M_{\theta}, M_{\theta}\right]$.

For each $\mathbf{x} \in \mathbf{X}$, and for every linked pair $i j \in g$ and state $\theta$, construct the agent's net transfer from others $\left(z_{i}(\theta), z_{j}(\theta)\right)$ as follows:

$$
z_{i}(\theta) \equiv \sum_{k \neq j: i k \in g} x_{i k}(\theta) \text { and } z_{j}(\theta) \equiv \sum_{k \neq i: j k \in g} x_{j k}(\theta)
$$

Now for each linked pair $i j \in g$ the bilateral norm allocates consumptions $c_{i}(\theta), c_{j}(\theta)$. Build $x_{i j}^{\prime}(\theta)$ by setting

$$
x_{i j}^{\prime}(\theta)=c_{i}(\theta)-y_{i}(\theta)-z_{i}(\theta)
$$

provided that the absolute value does not exceed $M(\theta)$. Otherwise, set $x_{i j}^{\prime}$ equal to $M(\theta)$ or to $-M(\theta)$ as the case may be. Denote by $\mathbf{x}^{\prime}$ the vector so generated; by construction, $\mathbf{x}^{\prime}$ also lies in $\mathbf{X}$. Let $\phi$ be the mapping that takes $\mathbf{x}$ to $\mathbf{x}^{\prime}$; obviously, $\phi$ continuously maps $\mathbf{X}$ into itself. By Brouwer's fixed point theorem, there is $\mathbf{x}^{*} \in \mathbf{X}$ such that $\phi\left(\mathbf{x}^{*}\right)=\mathbf{x}^{*}$, and the proof of existence is complete. 
It remains to prove that any fixed point yields strictly positive consumption to all individuals in all states. Suppose this is false for some fixed point $\mathbf{x}^{*}$. We know that in any connected component $d$ all consumptions cannot be negative, as aggregate income is positive. Hence, there exists a linked pair of agents $i j \in d$ and a state $\theta$ such that $c_{i}(\theta) \leq 0$ and $c_{j}(\theta)>0$. By our assumption, it must be that $i$ is making no transfer to $j$, and her output $y_{i}(\theta)>0$. So she must be making a transfer to some other set of individuals, say $K$. By the same logic, $c_{k}(\theta) \leq 0$ for all $k \in K$. Repeating this argument for all individuals in $K$ (and continuing on to other linked individuals if necessary), we see that ultimately we must encounter a pair of linked individuals - say $\ell m$ - such that $\ell$ makes a positive transfer to $m, c_{\ell}(\theta) \leq 0$, and $c_{m}(\theta)>0$. This is a contradiction.

8.2. 1-sparse networks. The clusters of a network $g,\left\{C_{1}, . . C_{M}\right\}$, are the largest fully connected subnetworks of $g$. Note that every node belongs to at least one cluster. A network $g$ is made of clusters connected by bridges if $C_{n} \bigcap C_{m}$ is empty or a singleton for all $n, m \in\{1, \ldots M\}$. That is, within components clusters are connected only through one individual, such singletons are called bridge-nodes.

OBSERVATION 1. A network is 1-sparse is and only if it is composed of clusters connected by bridges.

Proof. Take a network $g$ composed of clusters and bridges and denote as $C_{1}, . . C_{M}$ its clusters. Take any triple of agents $(i, j, k)$ such that $(i, j)$ and $(j, k)$ are connected in the network. If $i$ and $k$ belong to the same cluster $C$, then clearly $j$ does too. By definition of a cluster $i k \in g$ and $\ell(i, j, k)=3$. Now assume that $i$ and $k$ do not belong to the same clusters. In this case, it must be that $j$ is a node-bridge and, since there is no other path from $i$ to $j, \ell(i, j, k)=2$. As either way $\ell(i, j, k) \leq 3, g$ is 1 -sparse.

Now, assume that $g$ is not composed of clusters clusters connected by bridges. This implies that there exists two clusters $C_{n}$ and $C_{m}$ so that $C_{n} \bigcap C_{m}$ has more than one element. Hence, there is a triple of agents $(i, j, k)$ such that $i \in C_{n} \backslash C_{n} \bigcap C_{m}, k \in C_{m} \backslash C_{n} \bigcap C_{m}, i j \in g$ and $j k \in g, \ell(i, j, k) \geq 4$. This implies that $g$ sparseness is at least 2 .

8.3. Lemmas for the Characterization of $q$-Stability. Proof of Lemma 1. Let the bilateral norm generate a consistent consumption allocation for $g$. Now remove the link $i j$ in $g$ to obtain a subnetwork $g^{\prime}$, which is still connected. So there is a path of direct links connecting $i$ and $j$ via some ordered set of individuals $1, \ldots, p$, where $i=1, j=p$, and $(k, k+1) \in g^{\prime}$ for all $k=1, \ldots, p-1$.

Consider some transfer scheme $\mathbf{x}$ associated with the consistent consumption allocation on $g$. We construct a transfer scheme $\mathbf{x}^{\prime}$ on $g^{\prime}$ as follows. Fix some state $\theta$. For each $k \in\{1, \ldots, p-1\}$, define $x^{\prime} \equiv x_{k, k+1}(\theta)+x_{i j}(\theta)$. Set $x_{k \ell}^{\prime}(\theta)=x_{k \ell}(\theta)$ for every other pair of linked agents $k \ell$ in $g^{\prime}$. It is easy to see that for every linked pair $k \ell$, the sum of third-party obligations is unchanged over $\mathbf{x}$ and $\mathbf{x}^{\prime}: z_{k}^{\prime}(\theta)+z_{\ell}^{\prime}(\theta)=z_{k}(\theta)+z_{\ell}(\theta)$. Moreover, this scheme yields exactly the same consumption vector as before. Because the bilateral norm aggregates third-party obligations, it will indeed recommend the same consumption allocation for $g^{\prime}$ as it did for $g$. Therefore the earlier consumption allocation is still consistent.

The same argument can be extended to all connected subnetworks of $g$ by removing one link at a time. 
Proof of Lemma 2. Sufficiency. Suppose that there exists a triple of agents $i, j$ and $k$ with a shortest cycle of length greater than $q+2$. Let $j$ delete his link with $i$. Then the length of the path between $i$ and $k$ in the cycle is greater than $q$, and because the cycle is minimal, there is no other path between $i$ and $k$ of smaller size. But this implies that if one deletes all links to $j$ along paths from $j$ to $i$ of size smaller or equal to $q+1$, link $j k$ remains. In particular, this implies that $j$ remains linked to $i$ in the resulting graph, and remains linked (through $i$ ) to all agents who have severed their direct link to $j$. Hence, the number of components of the graph has not changed.

Necessity. Conversely, consider a link $i j$ for which the deletion of all links to $i$ along the paths to $j$ of size smaller than $q+1$ does not increase the number of components in the graph. This implies that $i$ and $j$ remain connected in the graph, so that there exists an agent $k$ directly connected to $i$, such that all paths from $k$ to $j$ have size greater than $q$. Then, for the tripe $k, j, i$, all cycles connecting the three agents must have length greater than $q+2$.

Proof of Corollary 1. Networks that have only trees as components are the only network that are 0 -sparse. Since stability under weak punishment corresponds to $q$-stability for $q=0$, the proof of the fist part of the corollary is a direct application of Proposition 3.

Take any network $g$. There exists $\bar{q}$ such that $g$ is $q$-sparse for $q \geq \bar{q}$. It follows from Proposition 3 that $g$ is universally $q$-stable under monotone transfer norms for $q \geq \bar{q}$. That is, the inequality (3) where $v_{i}\left(g^{\prime}\right)$ is determined by level $q$ punishment for $q \geq \bar{q}$ holds for every player $i$, every state $\theta$ and every set of direct neighbors $S$ of $i$. Under strong punishment, any individual receives a payoff $v_{i}^{*}(\emptyset)$ following a deviation. To be sure, $v_{i}\left(g^{\prime}\right) \geq v_{i}^{*}(\emptyset)$ for all $i$ and $g^{\prime}$. Hence, (3) holds under strong punishment too and $g$ is stable.

\subsection{Short-Term Effects.}

Proof of Proposition 4. Consider two networks $g$ and $g^{\prime}$ of same size $n$. Take a realization $\mathbf{y}$ for these networks. Denote as $\Pi(\mathbf{y})$ the set of permutations of $\mathbf{y}$. Call an individual a giver if $y_{i} \geq \sum_{j} y_{j} / n$ and a recipient otherwise. Let $\gamma(\mathbf{y})$ be the number of givers and $R(\mathbf{y})$ the set of recipients in $\mathbf{y}$. Let $i$ be a critical node of degree $\gamma$. We claim that among the income realizations in $\Pi(\mathbf{y})$, the tightest constraints is obtained for node $i$ when all agents in the subnetwork of size $\gamma$ originating at $i$ are givers. For all income realization $\mathbf{y}^{\prime} \in \Pi(\mathbf{y})$ the average income is the same, such that (5) is maximal for $i$ if it maximizes

$$
y_{i}^{\prime}+\sum_{j \mid x_{i j} \geq 0} x_{i j}\left(\mathbf{y}^{\prime}\right)
$$

To equalize the incomes a total transfer of $\tau(\mathbf{y}) \equiv \sum_{j \in R(\mathbf{y})}\left[\sum_{l} y_{l} / n-y_{j}\right]$ needs to be made from givers to recipients. Since $i$ is critical of degree $\gamma$, when all agents in the subnetwork of size $\gamma$ originating in $i$ are givers, all transfers to be made need to transit via her. In this case, (7) clearly takes its maximal value $\sum_{l} y_{l} / n+\tau(\mathbf{y})$ irrespective of $i$ 's particular income.

[The only other candidate would be a recipient $j$ who receives exactly $\tau$ in transfers. But then (7) would be lower since $y_{j}<\sum_{l} y_{l} / n$.]

Hence, for any realization in $\Pi(\mathbf{y})$, the tightest constraint will be the same in any decomposable network. To compute the exact threshold value of $\delta$ for which a decomposable network is stable, 
one needs to find the state $\mathbf{y}$ which results in the tightest constraint, i.e. that solves the problem:

$$
\max _{\mathbf{y}} u\left(\sum_{l} y_{l} / n+\tau(\mathbf{y})\right)-u\left(\sum_{l} y_{l} / n\right) .
$$

The solution $\mathbf{y}^{*}$ of this problem depends on the shape of the utility function. Once $\mathbf{y}^{*}$ is known, the threshold value can easily be computed.

Let $\mathbf{y}^{*}$ be the solution to the maximization problem (8). If the network $g^{\prime}$ has a critical node of degree $\gamma\left(\mathbf{y}^{*}\right)$ then clearly $g$ and $g^{\prime}$ have the same stability property. Otherwise, if $g^{\prime}$ does not have a critical node of degree $\gamma\left(\mathbf{y}^{*}\right)$, the consumption can be equalized in a way that the highest consumption a deviating agent could achieve when $\mathbf{y}^{*}$ is realized is strictly less than $\sum_{l} y_{l} / n+\tau(\mathbf{y})$ and the conclusion follows.

Proof of Corollary 2. Consider the binding constraint in graph $g$. Let $\theta$ be the corresponding realization of the shock, and $i$ the agent whose constraint is binding. Compute a transfer scheme for graph $g^{\prime}$ as follows. For any $\theta^{\prime} \neq \theta, \mathbf{x}\left(\theta^{\prime}\right)$ is the same for the two graphs. For $\theta$, one may be able to relax the transfer constraint, by using additional links. In other words, if new links permit a rerouting of the transfers to bypass $i, i^{\prime}$ s constraint will necessarily be relaxed. 


\section{RefEREnCES}

(1) Allen, F. and D. Gale (2000) "Financial Contagion," Journal of Political Economy 108(1), 1-33.

(2) Bandiera. O and I. Rasul (2002) "Social Networks and Technology Adoption in Northern Mozambique," STICERD - Development Economics Papers 35.

(3) Bala, V. and Goyal, S. (2000) "A Non-cooperative Model of Network Formation," Econometrica 68(5), 1181-1229.

(4) Bramoullé Y., and R. Kranton (2004) "Public Goods in Networks: how social networks can shape social learning and innovation," mimeo.

(5) Bramoullé Y., and R. Kranton (2005) "Risk-Sharing Networks," mimeo.

(6) Calvo-Armengol, T. and M. Jackson (2004) "The Effects of Social Networks on Employment and Inequality, American Economic Review, 94(3), 426- 454.

(7) Chatterjee, K. and S. Xu (2004), "Technology Diffusion by Learning from Neighbors," Advances in Applied Probability 36, 355-376.

(8) Coate, S. and M. Ravallion (1993) "Reciprocity without Commitment: Characterization and Performance of Informal Insurance Arrangements," Journal of Development Economics 40, 1-24.

(9) Conley, T.G. and Udry, C.R. (2003) "Learning about a New Technology: Pineapple in Ghana," BREAD Working Paper 012.

(10) Dearden, K., L. Pritchett and J. Brown (2004) "Learning From Neighbors: Social Learning About Child Feeding During Diarrheal Episodes," working paper.

(11) Dercon, S. and J. de Weerdt (2000) "Risk-Sharing Networks and Insurance against Illness," mimeo.

(12) Dutta B. and D. Ray (1989) "A concept of egalitarianism under participation constraints," Econometrica 57, 615-635.

(13) Fafchamps, M. (1992) "Solidarity Networks in Preindustrial Societies: Rational Peasants with a Moral Economy" Economic Development and Cultural Change 41(1), 147-74.

(14) Fafchamps, M. and S. Lund (2003) "Risk Sharing Networks in Rural Philippines," Journal of Development Economics 71, 261-87.

(15) Fafchamps, M. and F. Gubert (2004) "The Formation of Risk-Sharing Networks," mimeo.

(16) Farrell, J. and S. Scotchmer (1988) "Partnerships," Quarterly Journal of Economics 103 (2), 279-297.

(17) Freixas, X. , B. Parigi and J. C. Rochet (2000) "Systemic Risk, Interbank Relations, and Liquidity Provision by the Central Bank," Journal of Money, Credit and Banking 32(3), 611-638.

(18) Genicot, G. and D. Ray (2003) "Group Formation in Risk Sharing Arrangements," Review of Economic Studies 70, 87-113.

(19) Genicot, G. and D. Ray (2005) "Informal Insurance, Enforcement Constraints, and Group Formation," in Group Formation in Economics; Networks, Clubs and Coalitions, edited by G. Demange and M. Wooders, Cambridge University Press, Cambridge U.K.

(20) Goyal, S. and S. Joshi (2003) "Networks of Collaboration in Oligopoly," Games and Economic Behavior 43, 57-85.

(21) Herreiner D., A. Kirman, and G. Weisbuch (2000) Market Organisation and Trading Relationships, Economic Journal, 110, 411-436.

(22) Hoff, K. (1997) "Informal Insurance and the Poverty Trap," mimeo, University of Maryland. 
(23) Jackson M. (2003) "The Stability and Efficiency of Economic and Social Networks," in Networks and Groups: Models of Strategic Formation, edited by B. Dutta and M. Jackson, SpringerVerlag, Heidelberg.

(24) Jackson M. (2005) "A Survey of Models of Network Formation: Stability and Efficiency," in Group Formation in Economics; Networks, Clubs and Coalitions, edited by G. Demange and M. Wooders, Cambridge University Press, Cambridge U.K.

(25) Kimball, M. (1988) "Farmer Cooperatives as Behavior towards Risk," American Economic Review 78, 224-232.

(26) Kletzer, K. and B. Wright (2000) "Sovereign Debt as Intertemporal Barter," American Economic Review 90, 621-639.

(27) Kocherlakota, N. (1996) "Implications of Efficient Risk Sharing without Commitment," Review of Economic Studies 63, 595-609.

(28) Kranton, R. and D. Minehart (2000), "Competition for Goods in Buyer-Seller Networks," Review of Economic Design 5, 301-332.

(29) Kranton, R. and D. Minehart (2001), "A Theory of Buyer-Seller Networks," American Economic Review.

(30) Kremer M. and Miguel (2003) "Networks, Social Learning, and Technology Adoption: The Case of Deworming Drugs in Kenya," mimeo.

(31) Ligon, E., J. Thomas and T. Worrall (2002) "Mutual Insurance and Limited Commitment: Theory and Evidence in Village Economies," Review of Economic Studies 69, 115-139.

(32) Lippert, S. and G. Spagnolo (2005), "Networks of Relations," mimeo.

(33) Murgai, R., P. Winters, E. Sadoulet and A. de Janvry (2002) "Localized and Incomplete Mutual Insurance," Journal of Development Economics 67, 245-274.

(34) Platteau, J.-P. (2004) "Solidarity Norms and Institutions in Agrarian Societies: Static and Dynamic Considerations," forthcoming in the Hanbook on Gift-Giving, Reciprocity and Altruism, S. Kolm and J. Mercier-Ythier (eds.) Amsterdam: North-Holland and Elsevier .

(35) Posner, R. (1980), "A Theory of Primitive Society, with Special Reference to Law," Journal of Law and Economics 23, 1-53.

(36) Rochet, J.C. and J. Tirole (1996) "Interbank Lending and Systemic Risk," Journal of Money, Credit and Banking 28(2), 733-762.

(37) Stack C.B. (1974) All Our Kin: Strategies for Survival in Black Community, New York: Harper \& Row.

(38) Tesfatsion, L. (1997) "A Trade Network Game with Endogenous Partner Selection," in H. Amman et al (eds.), Computational Approaches to Economic Problems, Kluwer Academic Publishers, 249-269.

(39) Tesfatsion, L. (1998) "Gale-Shapley matching in an Evolutionary Trade Network Game," Iowa State University Economic Report no. 43.

(40) Vega-Redondo, V. (2003) "Building up Social Capital in a Changing World," mimeo.

(41) Wang, P. and A. Watts (2002), "Formation of Buyer-Seller Trade Networks in a QualityDifferentiated Product Market," mimeo., Southern Illinois University.

(42) de Weerdt, J. (2002) "Risk Sharing and Endogenous Network Formation," United Nations University Discussion Paper No. 2002/57.

(43) Wasserman, S. and Faust, K. (1994) Social network analysis: Methods and applications. New York: Cambridge University Press.

(44) Watts, D.J. and S.H. Strogatz (1998) "Collective Dynamics of 'Small-World' Networks," Nature 393, 440-42. 
(45) Wellman, B. (1992) "Which types of ties and networks provide which types of support?" In E. Lawler, Markovsky, B., Ridgeway, C., and Walker, H. (Ed.), Advances in Group Processes. Greenwich, CT, JAI Press. 